\title{
LA LEY DE BARRIOS EN CATALUÑA: \\ LA PERSPECTIVA DE GÉNERO COMO HERRAMIENTA DE PLANIFICACIÓN
}

\author{
ZAida MuXí Martínez y AdRiana CiOCOletTo \\ Universidad Politécnica de Cataluña
}

Recibido 09/02/2011

Aceptado 20/05/2011

\section{Resumen}

La Ley catalana 2 / 2004, de mejora de barrios, áreas urbanas y villas que requieren atención especial, ha sido una herramienta que permite comenzar a desarrollar un urbanismo con perspectiva de género. El punto 6 de la ley se refiere a la aplicación de la igualdad de oportunidades en el uso del espacio público y los equipamientos en la renovación urbana. Un análisis comparativo de una selección de las propuestas presentadas hasta la fecha, y un análisis in situ de los proyectos ya terminados permitirá obtener la evolución de la aplicación de la perspectiva de género en la planificación urbana. En resumen, esta investigación podría ser la oportunidad para revisar lo que se ha hecho y cómo, dando como resultado nuevas recomendaciones para las intervenciones junto a la aplicación de la perspectiva de género en la planificación urbana.

Palabras claves: Renovación Urbana. Perspectiva de género. Barrios. Vida cotidiana. Urbanismo Catalán. 


\begin{abstract}
The Catalonian Law 2 / 2004, improvement for districts, urban areas and villages that require special attention, has been a leading tool to start developing an urban planning with a gender perspective. The point 6 of the law is about the promotion of equal opportunities in the use of public facilities and public spaces by the means of urban renovation. A comparative analysis of a selection of the proposals submitted so far, and an on-site analysis of the projects already completed will permit to draw the evolution of the application of gender perspective on urban planning. Summing up, this research could be the opportunity to review what has been done and how, giving as result new recommendations for next interventions to be applying the gender perspective in urban planning.
\end{abstract}

Keywords: Urban renewall. Gender perspective. Neighbourhoods. Everyday life. Catalonian urbanism. 


\section{Introducción}

El siguiente artículo es parte del resultado del trabajo de investigación Recomendaciones para la aplicación de políticas de género en el urbanismo ${ }^{1}$, dentro del cual se realizaron estudios de casos, análisis bibliográfico y observación de la realidad para elaborar un documento de recomendaciones sobre el tema.

La ley 2/2004 del 4 de junio, es una ley autonómica aprobada por la Generalitat de Cataluña que tiene por objetivo intervenir en las mejoras de barrios, áreas urbanas y villas que requieren atención especial. La idea de esta ley, dotada económicamente en cada legislatura, es que cada ciudad determine los barrios que necesitan ser mejorados con el fin de evitar su progresiva guetización. La ley establece una serie de parámetros y criterios sociales, económicos, y físicos que se comparan localmente, permitiendo detectar estos barrios y justificar los proyectos que se presentan para optar a la subvención del 50\% del gasto total, que se cubre por el gobierno autonómico y que se han de desarrollar en cuatro años. Según la Ley, el barrio elegido ha de pertenecer a alguno de los tres tipos de tejido urbano determinados como áreas prioritarias de financiación: Áreas viejas y núcleos antiguos, Polígonos de viviendas y Áreas de urbanización marginal.

Los proyectos presentados deben incluir actuaciones en ocho campos, que serán verificados con un sistema de indicadores que se revisan y mejoran en cada convocatoria. Los puntos o campos de actuación propuestos por la ley son:

1. Mejora del espacio público y dotación de espacios verdes.

2. Rehabilitación y equipamientos de los elementos colectivos de los edificios

3. Provisión de equipamientos para el uso colectivo

4. Incorporación de las tecnologías de la información en los edificios

1. MuXí MarTineZ, Zaida (investigadora principal) Recomanacions per la implementació de polítiques de gènere al urbanisme. Entidad financiadora: Institut Català de les Dones, expediente U-62/06. Convenio de colaboración con la Universitat Politècnica de Catalunya grupo de investigación I-00868. 2006-2007. 
5. Fomento de la sostenibilidad del desarrollo urbano

6. Equidad de género en el uso del espacio urbano y de los equipamientos

7. Desarrollo de programas que comporten una mejora social, urbanística y económica del barrio

8. La accesibilidad y la supresión de barreras arquitectónicas

Una de las aportaciones más innovadoras de esta Ley es la incorporación de la perspectiva de género en una ley de rehabilitación urbana. El campo 6 establece la «equidad de género en el uso del espacio urbano y los equipamientos» como uno de los campos financiables que deben estar contemplados dentro de los proyectos de reforma urbana presentados por los ayuntamientos catalanes.

Para determinar si un área es de atención especial se utilizan cuatro series de parámetros o criterios que demuestran que está afectada por procesos de regresión urbanística y déficit de equipamientos y servicios; que sufre problemas demográficos; que hay problemas económicos, sociales o medioambientales y que sufren un déficit social y urbano. Quedando demostrados los criterios comparativamente con la ciudad en la que se halla, es decir, que no son parámetros absolutos sino relativos a cada situación urbana en particular.

La forma de presentación de los proyectos, incluye dos partes principales: una memoria diagnosis de la situación urbanística y estado de ejecución del planeamiento vigente y otra de la situación socioeconómica.

Para la redacción de los proyectos se cuenta con un manual orientativo para la redacción y elaboración de las propuestas según los 8 campos, que se revisa cada año mejorando y actualizando la información. La retroalimentación del conocimiento, de la teoría a la práctica, es otra de las características singulares de la gestión de la Ley con lo cual se está configurando un bagaje abierto y compartido de conocimiento.

Como en toda ley no es menor el contexto político en que es redactada, y en este caso merece que sea explicado. En el año 2004 el Gobierno autonómico es una coalición entre tres fuerzas políticas progresistas y en el cual el Instituto Catalán de las Mujeres (Institut Català de les Dones - ICD) depende directamente de la presidencia. Esta situación en el organigrama político generó una transversalidad de la cuestión de género en todas las políticas públicas. La transversalidad es una herramienta fundamental, imprescindible, para actuar con perspectiva de género, ya que significa entre otras cosas que no se considere a las mujeres como una minoría y un problema, sino como parte imprescindible y constitutiva de la sociedad y que para ello es necesario un redireccionamiento de los sistemas de pensamiento y actuación vigente que se 
basan en una neutralidad abstracta que corresponde a la experiencia y el pensamiento exclusivamente masculino. Por ello el hecho que el ICD dependiera de presidencia les permitió a las cuatro directoras ser parte activa del gobierno en todos los ámbitos, interviniendo y aportando desde la perspectiva de las mujeres saberes y conocimientos. En estas intervenciones está incluida la Ley de Barrios que aquí analizamos.

\subsection{Aplicación de la perspectiva de género en el proyecto urbano}

Es necesaria una mirada próxima y compleja sobre el espacio urbano, es decir, un planeamiento sin simplificación, en el que se hagan lecturas y análisis en diferentes escalas. Se trata de considerar las necesidades del mundo reproductivo de igual manera que las necesidades del mundo productivo. Se trata de equiparar las dos esferas poniéndolas en igualdad de valoración. El no tener en cuenta, como hasta ahora, las necesidades derivadas de las tareas asignadas al género femenino genera una descompensación de oportunidades y obligaciones que penaliza especialmente a las mujeres y a las personas dependientes como son las criaturas, las personas mayores o con falta de autonomía.

Para revertir esta situación es fundamental incorporar la participación de las mujeres en los procesos de reconocimiento, conocimiento y mejora de barrios, de manera que esta participación nos permita extraer de sus experiencias, derivadas del rol asignado cultural y tradicionalmente, un conocimiento directo de las realidades urbanas. Las mujeres somos las principales usuarias, disfrutadoras y sufridoras del espacio público y los equipamientos y en general en al cuidado de otros y otras.

La multiplicidad de actividades que llevamos a cabo y de las que somos responsables nos lleva a realizar recorridos de máximo aprovechamiento. Las mujeres realizamos una gestión del tiempo de máxima eficacia y para que esto sea posible se necesitan espacios urbanos complejos, que combinen usos (compras-escuelas-actividades cívicas y administrativas, etc.) al mismo tiempo que estén conectados con otras áreas de la ciudad por servicios de transporte público eficientes y variados.

El espacio público es el espacio conector de actividades dónde de forma individual o colectiva desarrollamos nuestra vida cotidiana y establecemos vínculos sociales y culturales. Por esto, desde una visión de género, el espacio público no es entendido como un espacio meramente funcional sino, como un espacio de experiencias vitales y por esto debe garantizar la igualdad de oportunidades y la equidad de usos. En este sentido, se deben procurar los usos mixtos que le den actividad, una movilidad sostenible que valore la proximidad y que priorice los desplazamientos peatonales, una accesibilidad 
sin discriminación, una percepción positiva de seguridad y una relación activa y entretejida con equipamientos, comercios y edificios en general. Como espacio de la memoria e identidad social, debe ser referente de las mujeres, reconociendo su protagonismo en la historia del barrio, pueblo o ciudad que en general es inexistente en los nomencladores de calles actuales.

Para equilibrar las responsabilidades, organizar las tareas reproductivas asignadas a cada género y proporcionar igualdad de oportunidades a mujeres y hombres, los equipamientos han de incorporar el «concepto de infraestructuras para la vida cotidiana» aportando nuevos espacios de actividades o equipamientos conforme la sociedad evoluciona. Una reflexión en torno a las experiencias diversas que se dan en la ciudad contemporánea, amplia el listado de necesidades, verifica que el espacio físico de los equipamientos debe proponer ámbitos para intercambiar, compartir, apoyar, integrar... y plantear «el tiempo de uso» como determinante para favorecer el acceso y la participación.

\subsection{Análisis de casos dentro de la ley}

En el contexto de esta ley nos interesa analizar qué ha significado, qué significa y puede significar el campo 6, equidad de género en el uso del espacio urbano y de los equipamientos, en los planteamientos de reformas urbanas, y de manera más amplia que significa la perspectiva de género aplicada al proyecto urbano.

Como hemos dicho el punto 6 es una aportación innovadora, que generó y genera no pocas dudas. Por ello a partir de las experiencias cada año se revisa y mejora el Manual para la elaboración de los proyectos de intervención integral en los barrios y áreas urbanas de atención especial y para la elaboración del informe de evaluación final que es una herramienta de gran ayuda dada la dificultad que presentan los ayuntamientos para realizar propuestas en este punto en concreto.

En las diferentes convocatorias se puede observar cómo han evolucionado las propuestas, cómo algunos equipos técnicos han adquirido nuevos conocimientos y han incorporado el género como herramienta de análisis y proyecto. Aún queda mucho por andar, pero los ejemplos que aquí analizaremos enseñan diferentes maneras de abordar un proyecto urbano con perspectiva de género.

En los primeros años de la convocatoria, la mayoría de actuaciones quedó acotada a las siguientes propuestas:

1. Actividades sociales: Talleres y campañas de concientización sobre el tema género. Mujeres inmigrantes e integración. 
2. Equipamientos para realizar las actividades anteriores.

3. Seguridad. Se refiere en su mayoría a mejorar la iluminación en zonas oscuras del espacio público.

La ampliación de los conocimientos y las mejoras en la aplicación de criterios de género en los proyectos han sido y son posibles gracias al soporte que las distintas administraciones (Generalitat, Diputación de Barcelona y Ayuntamientos) han dado a los trabajos de investigación de técnicas y técnicos, a la organización de jornadas, a la publicación de bibliografías y a la realización de cursos de formación técnica y ciudadana.

No es posible hoy día, febrero 2011, realizar una evaluación completa de los resultados de aplicación de estos criterios ya que de los 148 proyectos financiados por la Ley de barrios son pocos los que han finalizado de manera completa sus proyectos. Si bien en muchos de los barrios de la primera y segunda convocatoria se puede ya analizar provisionalmente la aplicación de la ley como por ejemplo, el Barrio de Santa Caterina en Barcelona y Can Anglada en Terrassa. También podemos comprobar que dentro de las últimas propuestas presentadas se intenta trabajar con mayor transversalidad aplicando los criterios de género en forma integral dentro del proyecto de reforma urbana.

Proponemos un análisis de tres casos desde la perspectiva de género en los que buscamos verificar si la aplicación del campo 6 mejora el espacio público y los equipamientos para la igualdad de género, y en un análisis transversal comprobar si las actuaciones en otros campos también se realizan desde la perspectiva de género. Los casos que hemos seleccionado para analizar plantean, de manera diferente a otros proyectos, la incorporación de la participación social como herramienta en el desarrollo del proyecto urbano.

\section{Estudio de casos}

\subsection{Barrio Collblanc / Torrassa, L'Hospitalet de Llobregat - (2004-2008)}

El barrio de Collblanc-Torrassa pertenece a la tipología de centro histórico, con alta densidad residencial de las décadas de los 50 s y 60s. Además de la falta de calidad de muchos de los edificios, la estrechez de las calles dificulta la movilidad interna y condiciona la calidad de los espacios públicos.

El «Proyecto equidad de género», impulsado por el Programa Municipal para la Mujer, es una propuesta que se ha planteado desde el área de políticas sociales con la intención que este programa sea la herramienta que permita la inclusión de los temas sociales y la perspectiva de género en los proyectos urbanos. Por ello se trabajó en fortalecer el tejido social a través del intercambio 
y el conocimiento mútuo. Uno de los primeros objetivos esenciales fue la creación de una asociación de mujeres del barrio, que a diferencia de otros barrios aquí no existía previamente.

La propuesta engloba seis programas de actuación:

- Coha: Servicio de intermediación para compartir vivienda

- Apropar (acercar): Participación social de mujeres inmigrantes

- Bellugant-nos (movernos): Actividad física y convivencia intercultural

- Participa: Potenciar la creación de una asociación de mujeres en el barrio

- Xarxa de dones (red de mujeres): Participación de mujeres del barrio en el proceso de planificación desde la perspectiva de género

- Estudi de mobilitat (estudio de movilidad): Movilidad adaptada a las necesidades de la vida cotidiana

Como característica del tejido social, el barrio está compuesto por aproximadamente un 30\% de su población de origen extracomunitario, consolidada en los últimos 6 años. Esto provoca en muchos casos problemas de convivencia en el uso de los espacios públicos, fundamentalmente por el desconocimiento y el temor al otro.

La percepción de la seguridad de las mujeres esta muy influida por este fenómeno. El centro de atención e información para la mujer, dentro del cual se encuentra el Proyecto de equidad de género, trabaja para promover el conocimiento entre las diferentes culturas y mejorar la convivencia.

A partir de este programa y con la asociación de mujeres formada, se ha realizado un informe sobre la percepción y demandas de mejoras de los espacios públicos, que se ha presentado al ayuntamiento para su consideración, del que destacan:

Mejora de los espacios públicos:

- Plantar árboles, situar bancos bajo la sombra de árboles y mejorar las zonas verdes existentes (Pl. Guernica)

- Percepción de inseguridad (Parc de la Marquesa)

- Limpieza en el barrio: ampliar la limpieza a todas las calles, no sólo a las principales.

Valoración de espacios para la práctica deportiva:

- Actualmente son insuficientes, debido cantidad de población y a la extensión territorial del distrito. 
- Abrir las instalaciones escolares para toda la población fuera del horario escolar, sin perder de vista la posibilidad, a más largo término, de construir instalaciones deportivas apropiadas.

- Proveer de servicio de guarderías o ludotecas en los equipamientos deportivos, para facilitar el acceso a la práctica deportiva de las mujeres con criaturas.

Local más adecuado para el grupo de mujeres: contar con un espacio céntrico y visible a los ojos de la ciudadanía, abierto al exterior, con la ventilación y la luz que actualmente no disponen.

El estudio de movilidad posiblemente sea el más próximo al proyecto urbanístico concreto. Realizado por el IERMB (Institut d'Estudis Regionals i Metropolitans de Barcelona) dirigido por Carme Miralles-Guash, con la colaboración de las investigadoras Gemma Solé Masó y Angel Cebollada Frontera. El trabajo, además del estudio sobre usos y percepción del espacio público y estrategias de movilidad, formula once propuestas de actuación, entre ellas:

- Garantizar la seguridad del espacio público con la presencia de la gente en la calle

- Políticas de prevención para evitar focos de inseguridad.

- En el diseño de los espacios públicos evitar zonas escondidas fuera de la vista de las personas y espacios generadores de inseguridad.

- Adaptar y ampliar los servicios públicos a las necesidades de la población actual.

- Elaborar el Plan de Movilidad Urbana con la máxima participación ciudadana.

- Mejorar la seguridad en estaciones de metro y vagones.

- Ampliar aceras, colocar barandillas en las calles y garantizar la iluminación y visibilidad.

Algunos aspectos como resultados de los criterios anteriores se pueden ver en el diseño de las calles que forman parte de la red de equipamientos y comercios donde se prioriza la movilidad peatonal y en la reforma del Parc de La Marquesa, donde se han instalado juegos para distintas edades y la instalación de un nuevo equipamiento cultural, en el centro del mismo, que le da vida al parque a distintas horas.

Dentro del proyecto de equidad de género se realizaron talleres participativos con mujeres del barrio para describir sus cotidianeidades y aportar conocimiento sobre el mismo desde la experiencia de las mujeres. Como derivado de esas sesiones se publicó el dossier Urbanismo y género, el papel de 

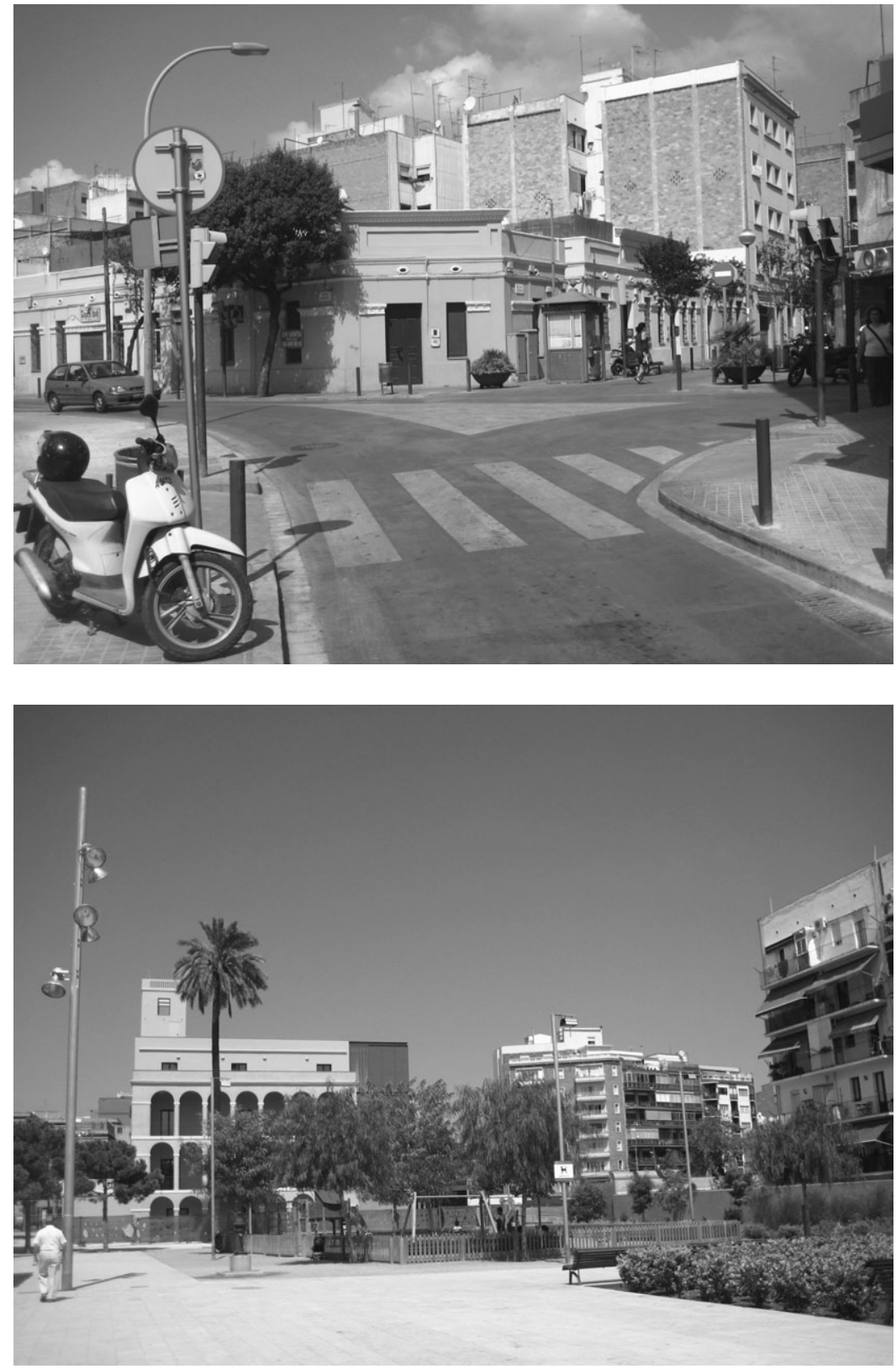

Feminismo/s 17, junio 2011, pp. 131-153 
las mujeres en la configuración de la ciudad² que tenía como objetivo acercar la reflexión urbana a todas las mujeres, ofreciendo una herramienta para la visibilización, información y capacitación técnica desde la perspectiva de género en el espacio urbano.

\subsection{Barrio de l'Erm, Manlleu - (2004-2008)}

La trama ortogonal con una sección de calle de gran amplitud y poca circulación vehicular es una de las características del barrio del Erm, en el municipio de Manlleu. A pesar de la gran amplitud de la calle, la distribución de aceras y calzada es desproporcionada, privilegiando el espacio vehicular. Otra característica del barrio, es la baja densidad y la sectorización de las tipologías edificatorias, las viviendas sociales en altura con espacios intermedios abandonados y viviendas unifamiliares en hilera. El barrio se encuentra rodeado por calles de mayor envergadura y de mucha afluencia vehicular que genera una frontera física con el resto de la ciudad.

La propuesta urbanística propone invertir radicalmente la relación de las calles-aceras favoreciendo el caminar y otros usos de los espacios públicos compartidos como el descanso y el encuentro, fomentando la vida en la calle.

La memoria del proyecto propone una visión de género integral y transversal que entrecruza cuatro temas principales: vivienda, equipamientos, espacio público y desarrollo socioeconómico.

La actuación en el barrio del Erm parte de la premisa de ser un laboratorio capaz de dar pautas para próximas rehabilitaciones del municipio, desde el comienzo se ha querido crear un proyecto muy conciente y enriquecedor, contando para ello con un equipo técnico experto en espacio público.

Para realizar la mejora del barrio se cuenta con la tutoría de equipos técnicos de la Diputación de Barcelona como soporte para los proyectos de la ley de barrios. Entre los materiales de difusión, información y formación de la Diputación de Barcelona se encuentra la Guía para el desarrollo de los proyectos de intervención integral en la que aparecen detalladamente los criterios de género a considerar en la realización de los proyectos arquitectónicos y urbanísticos.

Para una gestión más ágil, independiente y directa del proyecto urbanístico y del Plan de convivencia se ha creado el Instituto de desarrollo del Erm. De esta manera el proyecto de ejecución es el resultado del trabajo de un

2. MuXí MARTíneZ, Zaida en colaboración con COL·LECTIU PUNT 6; Urbanisme i Génere. El paper de les Dones en la configuració de la ciutat. De Dossier en Femení del programa municipal de la Dona de l'ajuntament de l'Hospitalet, Hospitalet del Llobregat, 2008. 
conjunto de expertos que suman sus experiencias y opiniones a las de las personas del barrio en un proceso de trabajo transversal.

De la propuesta urbanística destaca la visión integral de la remodelación en relación con el resto del municipio apostando claramente por los espacios públicos para la vida cotidiana de las personas definido en el documento «Estudio para la definición de criterios de urbanización en los procesos de renovación urbana».

El proyecto pone énfasis en las relaciones entre las calles y los edificios tanto públicos como de viviendas, haciendo un estudio y propuesta muy detallada de las tipologías de las calles del barrio. Se da mucha importancia a una clara diferenciación de las calles a través del carácter particular del diseño para identificarlas y que faciliten la orientación dentro del barrio.

\section{Los criterios de género específicamente detallados son:}

- En referencia a los espacios públicos la mejora de la movilidad, la accesibilidad y la permeabilidad. Prioridad peatonal y diseño de las aceras como espacio de encuentro. Rincones abiertos y sin obstáculos. Vegetación y mobiliario urbano que permita identificar las calles y que de esta manera ayude a reconocerlas y mejore la orientación de las personas. Espacios públicos con sol y sombra para distintos usos.

- En relación a las plantas bajas y los portales generar relaciones directas entre el espacio público y el espacio privado estudiando las secciones de calles y patios.

- Para mejorar la seguridad se propone eliminar barreras visuales y una iluminación estratégica diferenciando la de las calles y aceras, al tiempo que se potencia la actividad y la apropiación de la calle con el diseño del mobiliario.

Dentro de los proyectos ya ejecutados se encuentra una primera actuación en el colegio público Puig-Agut donde se ha eliminado el cerramiento perimetral formado por un muro y reemplazado por una reja. Esto ha permitido establecer una relación abierta entre el patio y el entorno como una manera de comenzar a concretar los criterios de permeabilidad, visibilidad y relación, ya que se ubica frente al mercado municipal del barrio.

Otro de los proyectos materializados dentro de los proyectos de espacios singulares, es la Plaza de San Antonio de Padua. La plaza ubicada entre dos bloques lineales de vivienda conformaba un patio con un gran desnivel hacia las calles por las cuales se accedía, generando escaleras y muretes que junto a la gran arboleda interior dificultaban uno de los objetivos generales de la 

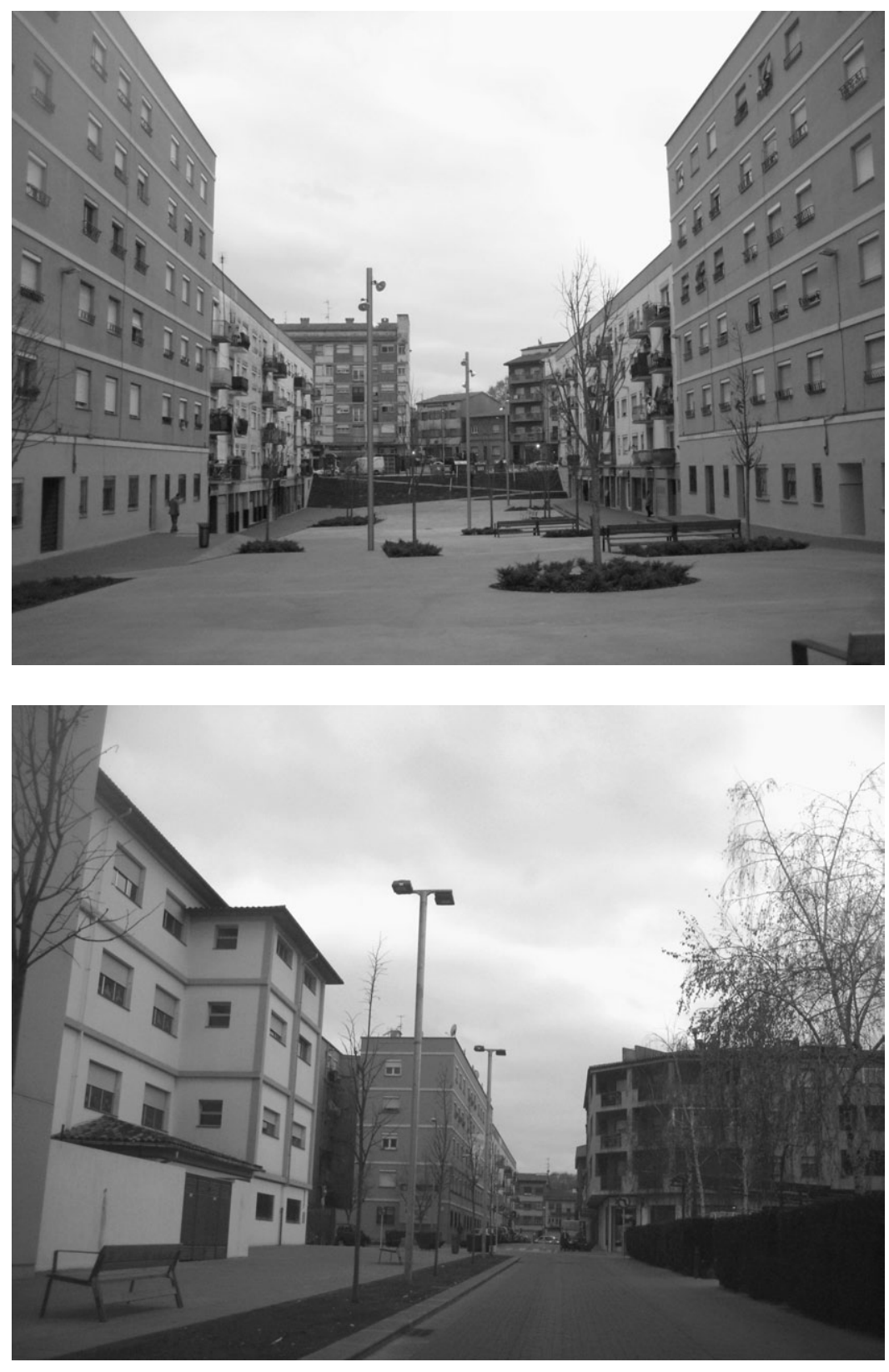

Feminismo/s 17, junio 2011, pp. 131-153 
remodelación que era la permeabilidad de los espacios públicos. Actualmente debido a la reforma, la plaza es un espacio con mucha visibilidad que facilita la accesibilidad al barrio y ha generado un espacio de relación más inclusivo ya que en general era utilizado mayormente por grupos de hombres.

Dentro de los proyectos generales está el cambio de secciones de calle las cuales algunas ya están ejecutadas favoreciendo la prioridad peatonal y el ensanchamiento de aceras con mobiliario e iluminación.

El programa social, «plan de convivencia», actúa paralelamente a la dinámica del proyecto urbano y está compuesto por cinco mesas de trabajo, fundamentalmente de educación, inclusión y participación ciudadana. Las mesas trabajan de forma interdisciplinaria y tienen la capacidad de interceder en las definiciones de los proyectos urbanísticos. Además, se mantiene constante diálogo con las personas del barrio sobre la evolución del proceso, que de esta manera está en constante replanteo y evaluación.

Uno de los trabajos realizados en el plan de convivencia es el sociograma del barrio que recoge la relación entre equipamientos, espacios públicos y usuarios. Esta información es una herramienta esencial para actuar en el espacio físico concretamente a través del conocimiento detallado de personas y dinámicas sociales.

\subsection{Barrio El Congost, Granollers - (2006-2010)}

El barrio del Congost en el municipio de Granollers provincia de Barcelona, se consolida en la década de los años 60 del siglo pasado con la llegada de la inmigración de otras zonas de España. Para dar respuesta a estas nuevas familias, se construyen masivamente viviendas públicas, muchas de ellas con una muy baja calidad constructiva y de habitabilidad. En la actualidad estas viviendas más deficientes están siendo habitadas por las nuevas familias inmigrantes, principalmente magrebíes y subsaharianas.

Otra de las características del barrio es su situación entre la margen del Río Congost y el trazado del ferrocarril, dificultando la relación y movilidad hacia los barrios vecinos. A esto se le agrega un gran déficit urbanístico por la falta o el mal estado de equipamientos y espacios públicos.

Dentro de los objetivos principales del proyecto de reforma se encuentra mejorar la accesibilidad general, los espacios públicos de relación y los equipamientos colectivos conjuntamente con programas sociales que favorezcan la inclusión, la convivencia y la igualdad de oportunidades. Además, se prevén programas de dinamización comercial para el fortalecimiento económico del barrio. 
En el caso del municipio de Granollers existe la regiduría de igualdad que es la encargada de llevar adelante el Plan para la igualdad de oportunidades. En el barrio del Congost, el plan es la herramienta utilizada para realizar diversas actividades para promover la participación del colectivo de mujeres, con el objetivo de que participen activamente en las mejoras urbanas en el marco de la aplicación del campo 6 de la ley de barrios.

Las actuaciones realizadas en orden cronológico fueron las siguientes:

- Diagnosis de género (datos segregados por sexo, edad y origen, elaboración de información cualitativa).

- Mesa de trabajo transversal: Presentación de informe impacto de género a todas las áreas implicadas en el proyecto de reforma del ayuntamiento.

- Formación de la comisión intercultural de mujeres del barrio del Congost: Grupos de discusión para detectar las necesidades de las mujeres del barrio (paralelamente al programa «Buen vecindario») y definir la estrategia y difusión del proceso de participación.

- Taller participativo «Las mujeres hacemos piña»: Organización de un encuentro para plantear problemáticas urbanísticas del barrio desde una perspectiva de género. Plenario y presentación de resultados ante el alcalde y la regiduría de igualdad.

- Devolución política (6 meses más tarde), con actuaciones en el territorio siguiendo criterios resultantes del taller participativo.

Para conocer la realidad multicultural de las mujeres del barrio fue necesario construir datos estadísticos por sexo, ya que como en la mayoría de los censos no estaban desglosados. Se trabajó con indicadores demográficos, socioeconómicos y de situación familiar. Esta informacion se completó con datos cualitativos obtenidos de entrevistas con distintos colectivos de mujeres ya agrupadas por nacionalidades (españolas, latinoamericanas, magrebies y subsaharianas), representantes de la escuela barrial y con personal técnico del ayuntamiento. Esta informacion permitió realizar un análisis previo con perspectiva de género.

Una primera aportación de las necesidades de las mujeres en relación a su experiencia cotidiana en el barrio surge del trabajo social realizado, por un lado los grupos de discusión interculturales, que crea la «Comisión intercultural de las mujeres del Congost» y por otro una primer experiencia de «Banco de tiempo».

La comisión trabajó conjuntamente con la regiduría en el plan de igualdad, estableciendo los criterios para realizar el taller participativo con el conjunto de mujeres del Congost. 


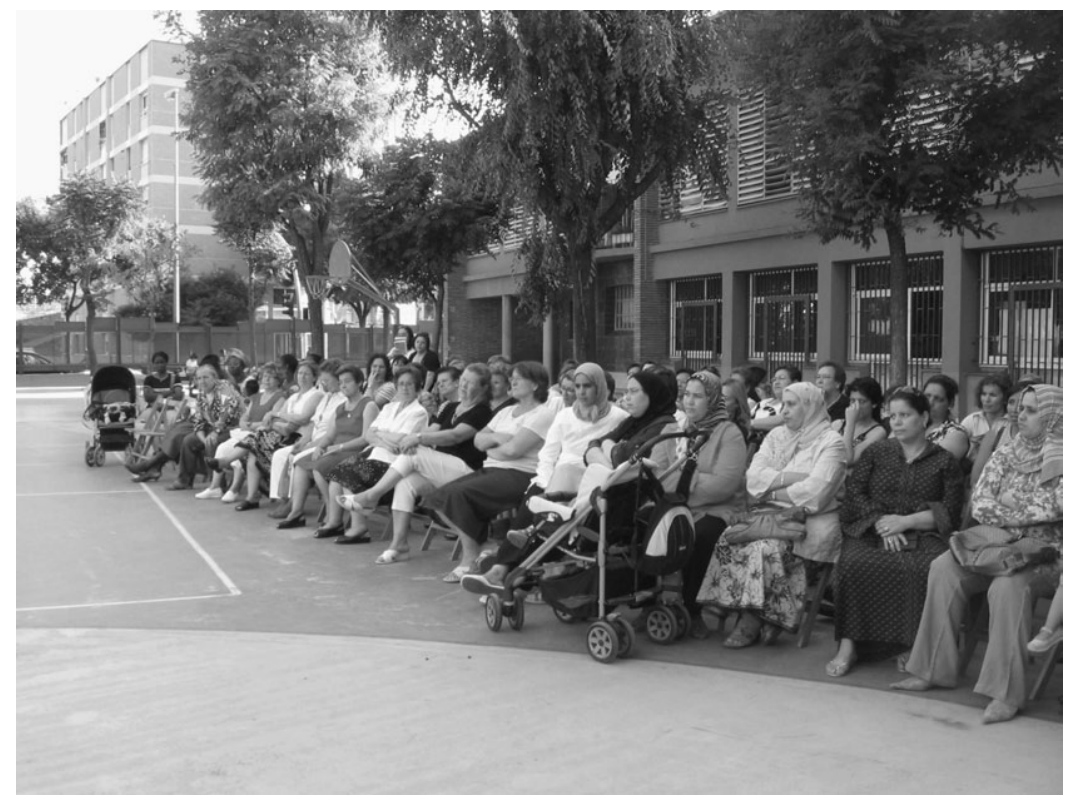

El primer encuentro «Las mujeres hacemos piña», consistió en una jornada intensiva donde asistieron un total de 99 mujeres acompañadas por 87 niñas y niños, para quienes se había previsto actividades y personal a cargo facilitando la participación de las madres. Del total de mujeres, 53\% pertenecían a Cataluña y resto de España, 23\% el Magreb, 17\% África Subsahariana y $7 \%$ Americana. ${ }^{3}$

El taller, dinamizado por el col-lectiu punt 6 , se centró en la problemática del urbanismo desde una perspectiva de género. El objetivo principal del taller fue ser capaces de nombrar, enumerar y argumentar la estructura de la vida cotidiana de las personas, hasta ahora infravalorada en el urbanismo como trabajo reproductivo ó como parte de un grupo minoritario de la población.

Como criterio de trabajo se visibilizó y reivindicó la experiencia de las mujeres como expertas cuando se habla de vida cotidiana en el entorno urbano, y en la necesidad de incluir este conocimiento empírico en el centro de

3. POLEO, Natàlia y SOLSONA, Glòria (2009) «La llei de barris: una oportunitat per avançar en la transversalitat de gènere i la participació de les dones. El cas del barri del Congost de Granollers» Col-lecció Grana número 26: Ciutats i Persones. Polítiques de gènere i participació ciutadana al món local Barcelona, Institut de Ciències Polítiques i Socials. 
las decisiones, de los procesos de diseño y de la planificación de las ciudades y del territorio.

De manera de poder profundizar en el análisis y sistematizar la información se realizaron grupos de discusión dinamizados que trabajaron en cuatro talleres temáticos: espacios públicos, seguridad, movilidad, y equipamientos públicos además de un taller-recorrido fotográfico de reconocimiento y valoración del barrio.

Algunos de los resultados elaborados con una lectura transversal entre sí reflejan la siguiente problemática:

- La plaza de la Libertad y su entorno fueron los espacios detectados como más problemáticos. La plaza por su diseño poco accesible y sobretodo los muros que segregan sectores y facilita la apropiación de algunos grupos de la población (hombres jóvenes) en detrimento de otros (mujeres e infantes). A las calles del entorno les falta mantenimiento, limpieza, iluminación adecuada, mobiliario y los árboles están deteriorados por incivismo.

- La percepción de inseguridad, además de lo comentado en el caso de la Plaza de la Libertad existe por la apropiación de los espacios públicos por grupos que se dedican a algunas actividades ilegales, edificios con viviendas vacías, el paso debajo del tren peligroso (túnel) al cual le falta iluminación y visibilidad.

- Los problemas de accesibilidad y movilidad son generalidades en todo el barrio con aceras y calles en mal estado; falta de rampas; paso de peatones no respetado; exceso de velocidad; falta transporte público hacia algunas zonas de concentración de trabajo; falta de carriles para bicicletas; falta de información en las paradas de autobuses. Esta problemática es más percibida en el entorno del colegio debido al uso de muchas familias del barrio.

- Los equipamientos reclamados fueron un pabellón deportivo, que existe pero está cerrado y en mal estado; la falta de espacio para que la juventud juegue al básquet $u$ otros deportes y la falta de una guardería municipal.

Actualmente entre las obras de mejora realizadas se encuentran:

- La mejora general de los espacios públicos atendiendo a los criterios de iluminación, visibilidad y accesibilidad destacando el entorno a la plaza de la libertad, acceso al colegio, a la estación de tren de cercanías y al túnel bajos las vías. 


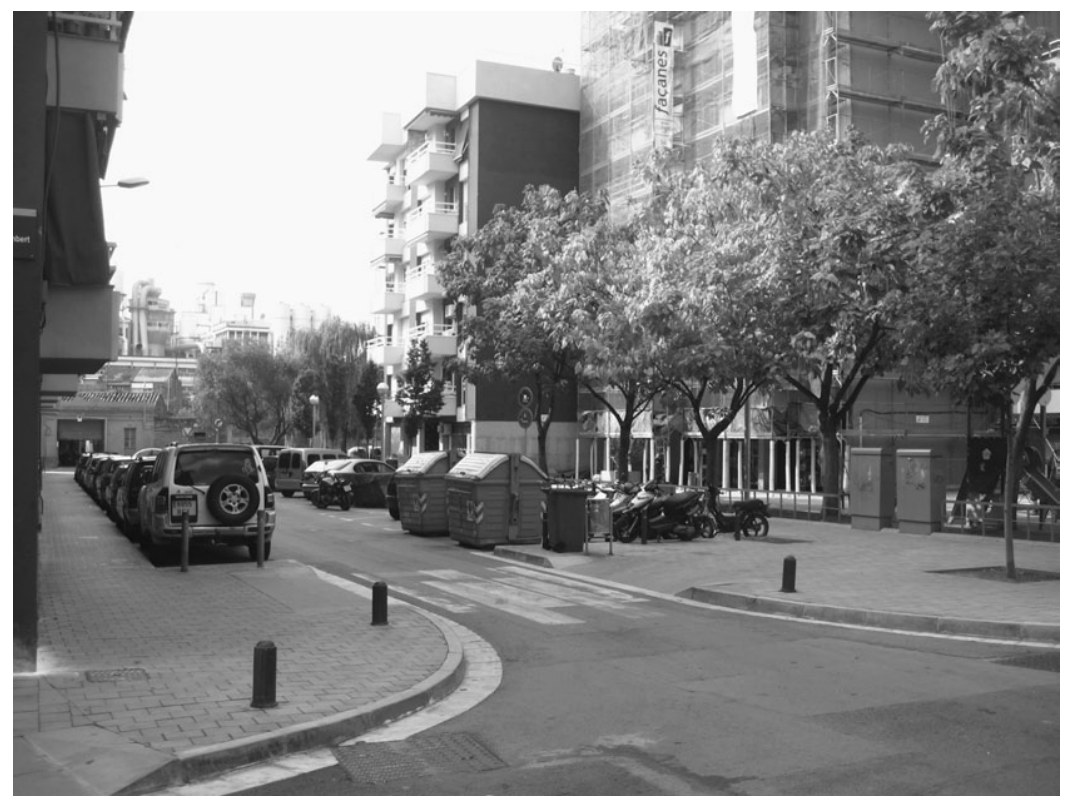

- La reforma de la Plaza de la Libertad atendiendo a los criterios de visibilidad y accesibilidad habiendo eliminado muretes y elementos que sectorizaban el uso de la plaza. Actualmente es un espacio compartido entre distintos colectivos del barrio principalmente para actividades de juegos infantiles de distintas edades y el descanso.

- La rehabilitación del equipamiento polideportivo además de revitalizar su uso anterior será un equipamiento con actividades sociales y culturales donde se mezclarán los distintos colectivos del barrio.

- La nueva guardería municipal ubicada frente al colegio completa la red de equipamientos reclamados por las mujeres.

- La reforma de la plaza de la Iglesia, abrió un nuevo espacio en relación al colegio y la guardería municipales además de mejorar la llegada a estos dos equipamientos desde otras zonas del barrio.

\section{Conclusiones sobre los proyectos analizados}

En los tres ejemplos estudiados aparece una combinación indispensable de programas de cohesión social paralelamente, aunque comenzado con anterioridad, al proyecto urbanístico. 

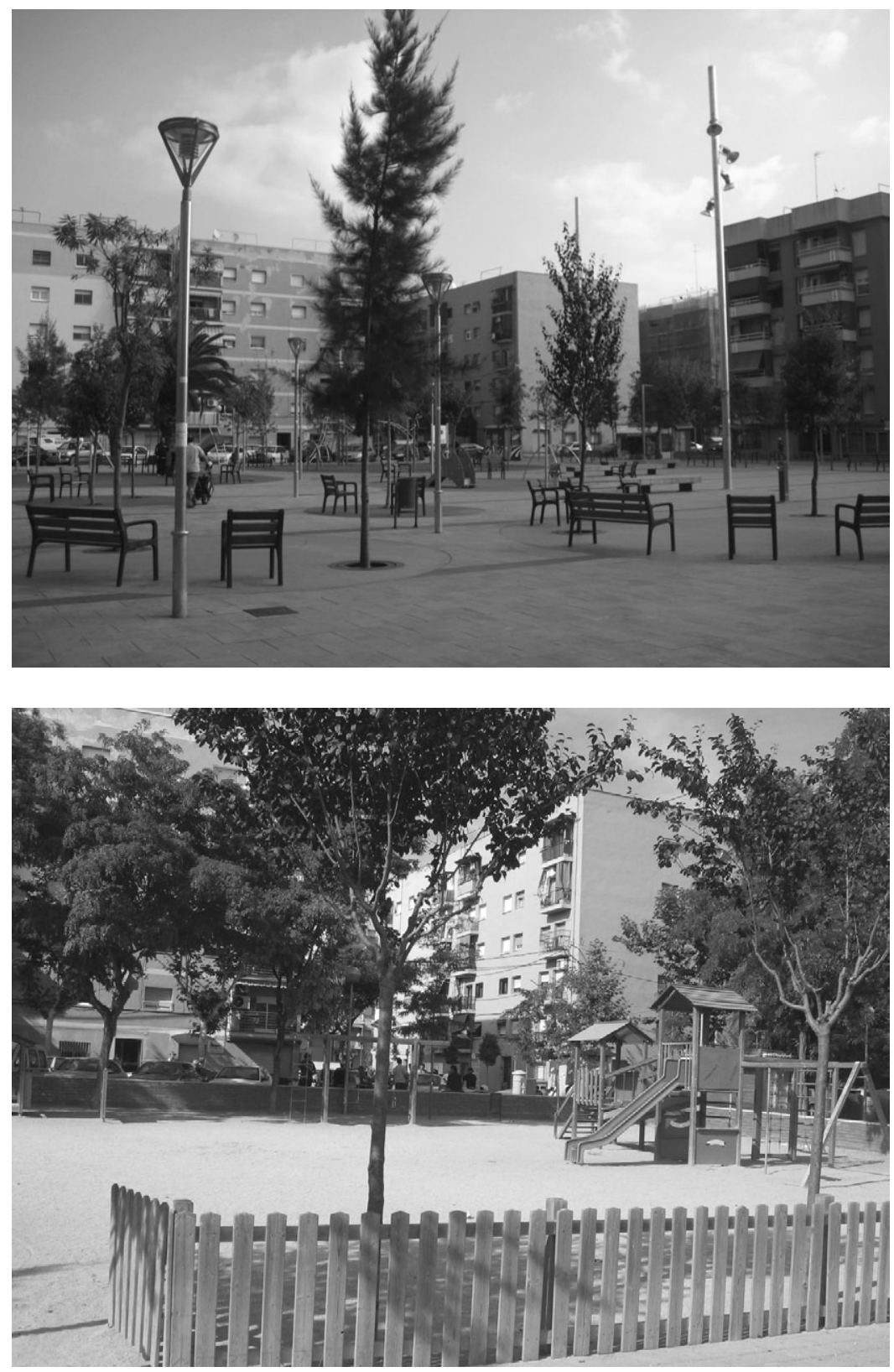

Feminismo/s 17, junio 2011, pp. 131-153 
Los programas sociales han avanzado formando o reforzando el tejido social que luego ha participado en la modificación de su propio entorno cotidiano. Debemos tener en cuenta que si bien todas las personas tenemos un conocimiento empírico de los espacios públicos, es necesario enseñar a participar y a valorar la experiencia cotidiana como conocimiento de una realidad determinada. La falta de visión integral entre los aspectos sociales y espaciales o urbanos, si es que se pueden separar, empobrecen los proyectos y les quita legitimidad.

En cuanto al campo 6 específicamente nos encontramos con un abanico muy diverso de metodologías para integrar a las mujeres de los barrios en la vida cívica y participativa, y, especialmente, para reforzar las redes de conocimiento y solidaridad entre ellas, asi como desvelar el valor de su experiencia como saber urbano.

Si bien las ejemplos analizados en mayor o menor medida integran a las mujeres en aspectos más directos del proyecto urbano, en un análisis más general de los barrios presentados hasta la convocatoria del año 2009, en su mayoría han entendido la participación de las mujeres y la perspectiva de género aplicados al proyecto urbano como cuestiones de integración social, de incorporar actividades lúdicas y formativas de «mujeres» (cursos de cocina, tejidos...) y de, en el mejor de los casos, construir un equipamiento para este fin. Actualmente, en la última convocatoria de la ley del año 2010, comienzan a aparecer propuestas más integrales como la transversalidad de género en el diseño, la ejecución y la evaluación de las actuaciones contenidas en los proyectos.

De lo analizado podemos extraer las siguientes consideraciones necesarias en relación al diseño de los espacios públicos, los equipamientos y la perspectiva de género:

- Realizar estudios detallados de las tipologías de calles que permiten realizar propuestas específicas no genéricas. En estos análisis es fundamental analizar las relaciones de permeabilidad que permiten las plantas bajas tanto en su diseño como por los usos que propone. Dar prioridad a las personas que van a pie, teniendo en cuenta que la unidad de trabajo es la barrial y que esta actuación tiene que reforzar la estructura interna. El espacio público se tiene que entender como espacio de relación, de encuentro.

- Integrar los equipamientos públicos a los espacios públicos de uso cotidiano. Permitir, por ejemplo, la permeabilidad visual de los patios de escuelas colabora en la seguridad en la calle, mejor rejas que muros 
ciegos. Generar ensanchamientos de acera en los accesos a los equipamientos públicos para generar espacios intermedios.

- Integrar los espacios con poca utilización o estancos a las redes de recorridos más utilizados. Reducir escaleras, desniveles y muretes que impidan la continuidad tanto de los peatones como de las visuales, garantizando todas las movilidades con la accesibilidad adecuada, con el uso intensivo y variado de los espacios públicos se consigue una percepción de seguridad.

- Integrar actividades diversas en los recorridos cotidianos de manera que el tiempo de los trayectos de desplazamiento sean utilitarios y seguros.

- Proyectar los equipamientos públicos teniendo máximas consideraciones a su permeabilidad visual y espacial con los espacios públicos circundantes.

- Hacer visibles las huellas que las actividades y las historias de las mujeres han dejado en la ciudad. Ya sea con la recuperación de espacios que antaño hubieran sido utilizados por las mujeres, como en muchos pueblos son los antiguos lavaderos, y también designar calles, plazas y equipamientos con nombres de mujeres.

En cuanto a la manera de analizar los barrios es imprescindible realizar estudios cualitativos con los que se obtiene información más detallada y sensible. Por ejemplo, formar grupos de trabajo integrado por vecinas del barrio capaces de evaluar el espacio público y los equipamientos de la red de usos cotidianos. Es fundamental incoporar estudios de la movilidad cotidiana que permitan saber como se utiliza el espacio público como conector de las diferentes actividades que las personas realizan.

Es importante tener en cuenta los datos estadísticos segregados por sexo además de edades y origenes e incoporar cuales son las cuestiones derivadas de la percepción de inseguridad que condicionan el libre movimiento de las mujeres por el barrio.

Incorporar la participación de las personas del barrio en las diferentes etapas del proyecto. El diagnóstico y diseño participativo son herramientas que pueden emplearse para conocer las dinámicas sociales del espacio público y para establecer criterios de diseño con las usuarias y usuarios. El programa de necesidades de los proyectos debería basarse en procesos de participación directa.

Incluirr la visión de los más pequeños, ya que junto a sus cuidadoras o cuidadores son de los que más utilizan el espacio público y necesitan consideraciones particulares y específicas. 
En relación a la gestión-coordinación, debe ser transversal y ágil, con un conocimiento directo del territorio en el que se trabaja para conocer las dinámicas sociales y la situación espacial existente. Muchas veces ni los conocimientos estadísticos, censales ni las planimetrías reflejan la complejidad de la realidad, especialmente los roles y usos diferenciados del espacio de los hombres y las mujeres.

El equipo técnico debe estar integrado por profesionales de diferentes disciplinas los cuales deben ser capaces de trabajar desde una perspectiva de género. Es indispensable el intercambio de conocimientos e información entre las diferentes áreas de actuación y que se articulen en una organización flexible que permita modificar rumbos o incorporar variaciones derivadas de la experiencia cotidiana en el lugar.

La evaluación del proyecto por parte del equipo técnico debe ser constante y también participada para construir un proceso vivo y no una fórmula cerrada.

La ley de barrios ha permitido la visibilización diferenciada de las necesidades de las mujeres en la ciudad y en los barrios. Aún falta para incorporar la cotidianeidad y las necesidades de la reproducción en igualdad de condiciones a aquellas derivadas de la producción, pero sin duda se ha iniciado un camino en el proyecto urbano que mejorará y cambiará las prioridades y proyectos urbanos futuros.

\section{Referencias bibliográficas}

AAVV;» La Carta Europea de la Mujer en la Ciudad, Bruselas» (Bèlgica). Experiència seleccionada en el Concurso de Buenas Prácticas patrocinado por Dubai en 1996.

AAVV; Urbanisme i gènere. Una visió necessària per a tothom. Barcelona, DIBA, Xarxa de Municipis, 2006.

BOFILl LeVI, Anna; Guia per al planejament urbanístic i l'ordenació urbanística amb la incorporació de criteris de gènere». Col•lecció Eines no 11 . Barcelona, Generalitat de Catalunya, Institut Catalá de les Dones, Departament de Política Territorial i Obres Públiques, 2008.

Bofill LeVI, Anna; Planejament urbanistic, espais urbans i espais interiors desde la perspectiva de les dones. Quaderns de l'Institut / 6. Barcelona, Generalitat de Catalunya, Institut Catalá de les Dones, Departament de Política Territorial i Obres Públiques, 2005.

Bоотн, Christine; «La participación de las mujeres en la planificación y el desarrollo urbanístico», en las jornadas del País Vasco organizado por la Diputación Foral de Bizkaia, gabinete del Diputado General. 
FAinstein, Susan y SERVON, Lisa (ed.); «Gender and Planning: A Reader. Journal of the American Planning Association, 2007.

GREED, Clara; «Genero y planificación del territorio. ¿Un mismo tema?» en el Fórum internacional de planificación del territorio desde una perpectiva de género, organizado por la Fundació Aurèlia Campany, 17 de noviembre de 1997.

Miralles-Guasch, Carme; Dones, mobilitat, temps i ciutats, col-lecció Quaderns de l'Institut 14, perspectives des del feminisme, Institut Català de les Dones, Barcelona, 2010.

MuXí MARTINEZ, Zaida (investigadora principal) Recomanacions per la implementació de polítiques de gènere al urbanisme. Entidad financiadora: Institut Català de les Dones, expediente U-62/06. Convenio de colaboración con la Universitat Politècnica de Catalunya grupo de investigación I-00868. 2006-2007.

MuXí MARTíNEZ, Zaida; «Barris per a viure» en Nous Horintzons. Els barris invisibles, $n^{\circ} 195$, editada per la Fundació Nous Horitzons, Barcelona, 2009.

MuXí MARTíNEZ, Zaida; «Infraestructures socials i de proximitat, equipaments per a la vida quotidiana» en la revista Quaderns d'Acció Social i Ciutadania. Revista d’informació, anàlisi i investigació socials, n³. Generalitat de Catalunya. Departament d'Acció Social i Ciutadania. Barcelona, (2008), pp. 60-62.

Muxí MartíneZ, Zaida; "Ciudad Pròxima. Urbanismo sin Género.» Revista Ingeniería y Territorio $\mathrm{n}^{\circ}$ 75, 2006. Revista del Colegio de Ingenieros de Caminos, Canales y Puertos, Barcelona.

Muxí MARTíneZ, Zaida; «Decir la ciudad desde la experiencia» Arquitectos, Construcción de la ciudad, n² 2, 2006. Revista de información del Consejo Superior de los Colegios de Arquitectos de España. Madrid.

MuXí MARTínEZ, Zaida; «El espacio no es neutro: reflexiones en torno al derecho a la vivienda y la ciudad desde una perspectiva de género» en Boletín Derecho a la vivienda y a la ciudad en América Latina, n 5. Editat per COHRE, 2008.

Muxí Martínez, Zaida; Urbanisme i Génere. El paper de les Dones en la confi-guració de la ciutat. De Dossier en Femení del programa municipal de la Dona del'ajuntament de l'Hospitalet, Hospitalet del Llobregat, 2008.

Muxí MARTíneZ, Zaida; «Ciutat Próxima. Una crítica feminista a la ciutat global» Sota les Llambordes, la platja, de José Miguel G.Cortés, editen H. Associació per a les Arts Contemporánies y Eumo editorial, Vic, Barcelona. 2008.

POLEO, Natàlia y SOLSONA, Glòria La llei de barris: una oportunitat per avançar en la transversalitat de gènere i la participació de les dones. El cas del barri del Congost de Granollers Col-lecció Grana número 26: Ciutats i Persones. Polítiques de gènere i participació ciutadana al món local. Barcelona, Institut de Ciències Polítiques i Socials.Barcelona, 2009.

SÁNCHEZ DE MADARIAGA, Inés; Urbanismo con prespectiva de género, Ed. Junta de Andalucía, Instituto Andaluz de la Mujer. (1998). 